OPEN ACCESS

Edited by: Jessica Duis,

Children's Hospital Colorado

United States

Reviewed by:

Daniela Francesca Giachino,

University of Turin, Italy

Giulio Piluso,

University of Campania Luigi Vanvitelli,

Italy

*Correspondence:

Rong Liu

liurong201305@sina.com

Xiaobo Chen

xiaobochen6362@163.com

tThese authors have contributed equally to this work

Specialty section:

This article was submitted to Genetics of Common and Rare

Diseases,

a section of the journal

Frontiers in Genetics

Received: 26 November 2019

Accepted: 31 July 2020

Published: 21 August 2020

Citation:

Song F, Feng S, Shen X, Du M, Yin H, Liu R and Chen X (2020)

Next-Generation Sequencing Revealed Disease-Causing Variants in Two Genes in a Patient With

Combined Features of Spherocytosis and Antley-Bixler Syndrome With Genital Anomalies and Disordered

Steroidogenesis.

Front. Genet. 11:976.

doi: 10.3389/fgene.2020.00976

\section{Next-Generation Sequencing} Revealed Disease-Causing Variants in Two Genes in a Patient With Combined Features of Spherocytosis and Antley-Bixler Syndrome With Genital Anomalies and Disordered Steroidogenesis

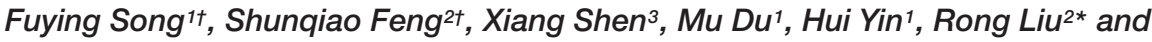 \\ Xiaobo Chen ${ }^{1 *}$ \\ ${ }^{1}$ Department of Endocrinology, Capital Institute of Pediatrics, Beijing, China, ${ }^{2}$ Department of Hematology, Capital Institute \\ of Pediatrics, Beijing, China, ${ }^{3}$ Running Gene Inc., Beijing, China
}

Conventionally, patients with combined rare diseases are often difficult to diagnose. This is because some clinicians tend to consider the multiple disease symptoms as the presentation of a complicated "syndrome." This pattern of thinking also confines their way of filtering pathogenic mutations. Some real pathogenic mutations might be ignored due to not covering all disease presentations. Here we report the case of a girl who was suffering from spherocytosis and Antley-Bixler syndrome with genital anomalies and disordered steroidogenesis. She remained undiagnosed even after targeted gene detection before. However, after performing next-generation sequencing and analyzing the sequencing data, we identified two mutations: c.2978T > A in ANK1 and c.1370G > A in POR. Our findings and experiences in diagnosing these mutations could contribute to the existing knowledge on the clinical and genetic diagnosis of patients with disease presentations in multiple systems.

Keywords: combined disease symptoms, multi-system involved, congenital adrenal hyperplasia, hereditary spherocytosis, POR, ANK1

\section{INTRODUCTION}

Hereditary spherocytosis (OMIM 182900), with a prevalence of $1 / 2,000$, is a hemolytic disorder characterized by the presence of spherical erythrocytes on a peripheral blood smear (Gallagher, 2005; Perrotta et al., 2008; Yocum et al., 2012). Clinical presentations include anemia, jaundice, and splenomegaly with heterogeneous severity (Perrotta et al., 2008). Genes related to hereditary spherocytosis include ANK1, SLC4A1, SPTB, EPB42, and SPTA. Among them, ANK1 is the most common disease-causing gene (Yocum et al., 2012). The ANK1 gene (OMIM*612641) encodes 
human erythroid ankyrin containing 1,880 amino acids. It combines tetramers of spectrin with the cytoplasmic domain of band three to form a spectrin-actin based membrane skeleton. This skeleton further interacts with the plasma membrane to stabilize the erythrocyte membrane (Gallagher, 2005). Defects in ankyrin synthesis or structure might result in instability of the erythrocyte membrane, thus causing hereditary spherocytosis.

Congenital adrenal hyperplasia $(\mathrm{CAH})$ is a group of autosomal recessive disorders characterized by impaired cortisol biosynthesis due to deficiencies in the various enzymes in the adrenal steroidogenesis pathway (Hannah-Shmouni et al., 2017; El-Maouche et al., 2017; Witchel, 2017). The candidate genes include CYP21A2, CYP11A1, CYP11B1, CYP17A1, HSD3B2, StAR, and POR (El-Maouche et al., 2017; Hannah-Shmouni et al., 2017). CAH due to POR deficiency is a rare variant of CAH. POR (OMIM*124015) encodes cytochrome P450 oxidoreductase. It is a flavoprotein that donates electrons to all microsomal $\mathrm{P} 450$ enzymes, including $17 \mathrm{OH}$ and $21 \mathrm{OH}$. Therefore, combined $17 \mathrm{OH}$ and $21 \mathrm{OH}$ deficiency could be observed in the patients (ElMaouche et al., 2017). The female patients with POR deficiency always present prenatal virilization, as the alternative pathway of producing androgens is not blocked (Arlt et al., 2004). Besides, some of these patients present skeleton malformations, such as craniosynostosis, radioulnar, and midface hypoplasia. This skeleton malformation presentation involved a form of $\mathrm{CAH}$ called Antley-Bixler syndrome or Antley-Bixler syndrome with genital anomalies and disordered steroidogenesis (OMIM 201750) in OMIM.

Herein, we report a Chinese patient presenting combined features of $\mathrm{CAH}$ and Antley-Bixler syndrome. Deficiencies in two related genes - ANK1 and POR - were identified by wholeexome sequencing. To our knowledge, patients carrying multiple causative gene mutations are uncommon since most patients are diagnosed as having a "syndrome" when they have symptoms involving multiple systems. Here, we share our experience in the diagnosis of this rare case.

\section{CASE PRESENTATION}

\section{Subject and Clinical Features}

The study was approved by the ethics committee of the Capital Institute of Pediatrics. Written informed consent was obtained from the patient's parents for the publication of this report and any accompanying images.

The patient was admitted to our hospital due to CAH. The patient was a female aged 10 years and six months. She was the first child born to non-consanguineous parents. She was born by normal cesarean delivery with a birth weight of 3,200 $\mathrm{g}$ (P25-P50) and a birth height of $51 \mathrm{~cm}$ (P50-P75). Her mother was diagnosed with polycystic ovary syndrome during pregnancy. Our patient presented clitoromegaly at birth with chromatosis of the vulva but not the skin. An ovarian cyst with a size of $5.7 \times 3.7 \times 4.3 \mathrm{~mm}$ was observed on the patient's right ovary when performing a pelvic ultrasound 23 days after birth. A karyotype test showed a normal karyotype of 46 autosomal chromosomes and two sex chromosomes.
A biomedical test indicated slight abnormality with increased $\mathrm{K}+(5.7 \mathrm{mmol} / \mathrm{l})$ and decreased $\mathrm{Na}+(133.9 \mathrm{mmol} / \mathrm{l})$. The concentration of the adrenocorticotropic hormone (ACTH) was $3.09 \mathrm{pg} / \mathrm{ml}$ and cortisol was $4.4 \mu \mathrm{g} / \mathrm{dl}$ at $8 \mathrm{am}$. In a sex hormone test, the luteinizing hormone $(\mathrm{LH})$ was $38.9 \mu \mathrm{l} \mathrm{U} / \mathrm{ml}$, the follicle-stimulating hormone (FSH) was $7.71 \mathrm{U} / \mathrm{l}$, testosterone (T) was $123.9 \mathrm{ng} / \mathrm{dl}$, and estradiol (E2) was $36.5 \mathrm{pg} / \mathrm{dl}$. When the patient was three years old, she had a clitoridectomy. At the age of five, the patient received gene detection by a gene panel in another hospital. However, the gene result revealed no possible pathogenic mutations (the original samples and results are missing). An ACTH stimulation test identified cortisol $(10.5 \mu \mathrm{g} / \mathrm{dl})$, Testosterone (16.4 $\mathrm{ng} / \mathrm{dl})$, and 17-OHP $(19.050 \mathrm{ng} / \mathrm{ml})$. Hydrocortisone $(5 \mathrm{mg} / \mathrm{bid})$ has been used to treat the patient until present, although no significant progress has been observed. When the patient was 5 years and 6 months old, she was admitted to our hospital due to "fever, fatigue, and pale skin lasting more than 1 week." She was found to have hepatosplenomegaly during a physical test, her liver was palpable at $2 \mathrm{~cm}$ and her spleen was palpable at $4 \mathrm{~cm}$ below the costal margin. In a routine blood test, her white blood cell count $(\mathrm{WBC}$ ) was $13.67 \times 10 \mathrm{E} 9 / 1$, hemoglobin (Hb) was $49 \mathrm{~g} / \mathrm{l}$, and reticulocyte (Ret) was 0.065. A blood smear revealed that the patient's proportion of spherocytes was $7 \%$ and seven nucleated red blood cells per 100 white blood cells could be counted. In an erythrocyte osmotic fragility test, hemolysis began at $0.56 \%$ salt solution (normal control at $0.42 \%$ ) and complete hemolysis began at $0.32 \%$ salt solution (normal control at $0.22 \%$ ). In liver function tests, total bilirubin (TB) was $30.9 \mu \mathrm{mol} / \mathrm{l}$, direct bilirubin (DB) $12.1 \mu \mathrm{mol} / \mathrm{l}$, and lactate dehydrogenase $(\mathrm{LDH}) 382 \mathrm{U} / \mathrm{l}$. A routine bone marrow test showed significantly active bone marrow proliferation and active erythroid proliferation, as well as normal folic acid, vitamin B12, ferritin, glucose phosphate isomerase, glucose-6phosphate dehydrogenase, and pyruvate kinase tests. Moreover, Coomb's test showed negative results. The patient was then clinically diagnosed with hereditary spherocytosis. The patient was then not treated and her jaundice relapsed. She received an ultrasound-guided percutaneous cholecystolithotomy and partial splenic artery ligation when she was 10 years old. The patient's condition soon improved and her jaundice did not return.

\section{Physical Examination During Admission}

The patient's weight was $29 \mathrm{~kg}$ (P25-P50) and her height was $144.5 \mathrm{~cm}$ (P25-P50). Her blood pressure was 100/60 mmHg and her bone age was normal, as was her mental status. A café-aulait spot with a diameter of $2 \mathrm{~cm}$ was observed at the patient's waist. She was in Tanner stage I of puberty. Arachnodactyly with long and slender fingers (Figure 1a) and toes (Figure 1b) was observed. The fourth and fifth metacarpal bones of the patient's hands were short and thick. Stiffness was observed in the elbow and knee joints. The patient could not make a fist due to joint contracture. Bony swelling was observed on the patient's sacrum.

\section{Imaging Tests}

X-ray examination: foot AP axial view: the bilateral fourth and fifth metatarsal bones of the patient were short, the fourth 


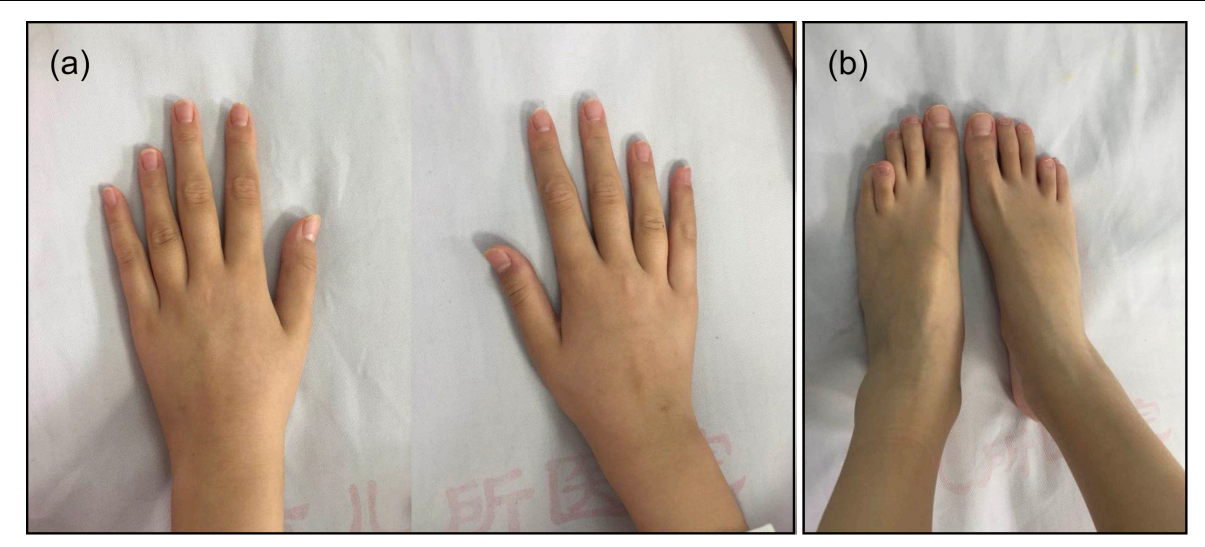

FIGURE 1 | Arachnodactyly with long and slender fingers (a) and toes (b) could be observed. The bilateral fourth and fifth metacarpal bones and metatarsal bones were all short.

phalanxes were short, and the bone mineral density of both feet was relatively low. Knee AP view: spiking of the bilateral tibial intercondylar eminence was observed. Lumbar spine AP view: slight scoliosis. Elbow AP view: mean trabecular plate density was relatively low. Contrast-enhanced CT scanning: no obvious abnormality was observed in the adrenal glands. However, the intrahepatic bile duct expansion, common bile duct expansion, and splenomegaly were observed.

\section{Laboratory Analyses}

Auxiliary examinations: the patient had normal serum electrolytes, while a sex hormone test showed that her FSH was $3.23 \mathrm{IU} / \mathrm{l}$, her $\mathrm{LH}$ was less than $0.1 \mathrm{IU} / \mathrm{l}$, and her E2 was less than $18.35 \mathrm{pmol} / \mathrm{l}$. She had testosterone (T) of $559 \mathrm{ng} / \mathrm{ml}$ (14-76), prolactin (PRL) of $28.61 \mathrm{ng} / \mathrm{ml}$, progesterone (PROG) of $0.25 \mathrm{nmol} / \mathrm{l}$, and parathyroid hormone (PTH) of $14.5 \mathrm{pg} / \mathrm{ml}$. Her 17-hydroxyprogesterone (17-OHP) was $2.14 \mathrm{ng} / \mathrm{ml}$, her dehydroisoandrosterone (DHEA) was $0.8 \mathrm{ng} / \mathrm{ml}$, her androstenedione was less than $0.3 \mathrm{ng} / \mathrm{ml}$, and her dihydrotestosterone was $13.86 \mathrm{pg} / \mathrm{ml}$. Her cortisol at $8 \mathrm{am}$, $4 \mathrm{pm}$, and 0 am were $2.25,4.9$, and $15.71 \mu \mathrm{g} / \mathrm{dl}$, respectively. Her renin, angiotensin, 1-angiotensin, and 2 -aldosterone were $3.08 \mathrm{ng} / \mathrm{ml}, 37 \mathrm{pg} / \mathrm{ml}, 40 \mathrm{pg} / \mathrm{ml}$, and $132 \mathrm{pg} / \mathrm{ml}$, respectively at recumbent position, and $3.69 \mathrm{ng} / \mathrm{ml}, 23 \mathrm{pg} / \mathrm{ml}, 37 \mathrm{pg} / \mathrm{ml}$, and $282 \mathrm{pg} / \mathrm{ml}$, respectively at erect position. Uterus and ovaries ultrasound images demonstrated that the length of the patient's uterus was $1.65 \mathrm{~cm}$ with a diameter of $0.5 \mathrm{~cm}$, and her cervix length was $1.6 \mathrm{~cm}$. The patient's endometrium could not be delineated clearly, not excluding the possibility that a primordial uterus existed.

\section{Treatment}

An ultrasound-guided percutaneous cholecystolithotomy and partial splenic artery ligation were performed. After the operation, the intrahepatic bile duct and common bile duct were expanded, the distal common bile duct narrowed, diffuse enlargement was observed, and the middle and lower parts of the spleen were infracted. Hydrocortisone $(5 \mathrm{mg} / \mathrm{bid})$ was taken orally by the patient for 6 days until she was discharged.

\section{METHODS}

\section{Whole-Exome Sequencing}

Proband's DNA was sequenced to discover the causal gene. Nextgeneration sequencing was performed by Running Gene Inc. following the manufacturer's protocol. DNA was isolated from peripheral blood using a DNA Isolation Kit (Bioteke, AU1802) and $1 \mu \mathrm{g}$ genomic DNA was fragmented into 200-300 bp lengths using a Covaris Acoustic System. The DNA fragments were then processed by end-repairing, A-tailing and adaptor ligation, a four-cycle pre-capture polymerase chain reaction (PCR) amplification, and targeted sequences capture. Captured DNA fragments were eluted and amplified by 15-cycle postcapture PCR. The final products were sequenced with $150 \mathrm{bp}$ paired-end reads on an Illumina NovaSeq platform according to the standard manual.

The raw data converted by NovaSeq were filtered and aligned against the human reference genome (hg19) using the BWA Aligner ${ }^{1}$. The single-nucleotide polymorphisms (SNPs) were called by using the Genome Analysis ToolKit (GATK) software ${ }^{2}$. Variants were annotated using $\mathrm{ANNOVAR}^{3}$. The effects of single-nucleotide variants (SNVs) were predicted by the SIFT, Polyphen-2, and MutationTaster programs. All variants were interpreted according to the standards for the interpretation of sequence variations recommended by ACMG (American College of Medical Genetics and Genomics) and categorized as pathogenic, likely pathogenic, variants of unknown clinical significance (VUS), likely benign, or benign. The associated phenotypic features of candidate genes were analyzed against the patient's phenotype. Core phenotypes were extracted and used to acquire a gene list of the virtual panel by the OMIM database ${ }^{4}$ and Mingjian (211.149.234.157/login). Re-annotation was conducted according to the virtual panel.

\footnotetext{
${ }^{1}$ http://bio-bwa.sourceforge.net/

${ }^{2}$ https://software.broadinstitute.org/gatk/

${ }^{3}$ http://annovar.openbioinformatics.org/en/latest/

${ }^{4}$ http://omim.org/
} 


\section{Sanger Sequencing}

The candidate causal genes discovered via whole-exome sequencing (WES) were confirmed by Sanger sequencing, and co-segregation analyses among the family were also conducted. The primers were designed using Primer Premier 5.0 (Premier Biosoft). PCR was conducted to amplify the fragments covering the mutated sites. The PCR products were further purified with a Zymoclean PCR Purification Kit and then sequenced with an ABI 3730 DNA Sequencer. The Sanger sequencing results were analyzed by Chromas Lite v2.01 (Technelysium Pty Ltd., Tewantin, QLD, Australia).

\section{RESULTS}

A heterozygous mutation (c.2978T > A) in ANK1 (NM_000037) and a homozygous mutation (c.1370G $>\mathrm{A}$ ) in $P O R$ (NM_000941) were identified by next-generation sequencing (Supplementary Figure S1), which resulted in amino acid alterations p.I993N and p.R457H, respectively. According to validation by Sanger sequencing in the proband's family, the proband's parents were both carriers of heterozygous mutation c.1370G $>\mathrm{A}$ in the $P O R$ gene. Neither parent had mutation c. $2978 \mathrm{~T}>\mathrm{A}$ in ANK1 (Figure 2).

According to the results, variant c.2978T $>\mathrm{A}$ in ANK1 was proven to be a de novo mutation by Sanger sequencing (PS2). This variant was also absent in the control (PM2) and is predicted to be deleterious by multiple in silico algorithms (PP2): it was predicted to be disease-causing by MutationTaster $(>0.99)$, deleterious by PROVEAN (-6.85), damaging by SIFT (0.00), and probably damaging by Polyphen-2 (1.000). The patient's phenotype was also specific for the disease, as she presented spherocytosis (PP4). Therefore, according to ACMG standards, this variant could be interpreted as likely pathogenic. Mutation c.1370G > A is a previously established pathogenic variant (PS1), and there is well-established functional studies supported the damaging effect of this variant (PS3). For instance, this mutation is classified as pathogenic according to ACMG guidelines.

\section{DISCUSSION AND CONCLUSION}

Hereditary spherocytosis is a common congenital hematologic disorder and also a common reason for inherited chronic hemolysis in Western countries. Hereditary spherocytosis has heterogeneous clinical presentations. In most cases, patients are asymptomatic or only present anemia. However, for patients have mild disease courses, $20-30 \%$ of these patients have mild splenomegaly and reticulocytosis, while for patients have moderate disease courses, $60-70 \%$ of them typically presented asymptomatic anemia, splenomegaly, and jaundice during infections in their childhood (Perrotta et al., 2008).

The structure of the erythrocyte membrane contains band-3 protein; protein 4.1; protein 4.2; ankyrin; spectrin; glycophorin A, B, C, and D; Rhesus (Rh) complex; CD47; LandsteinerWiener glycoprotein; dematin; tropomyosin; adducin; and tropomodulin. Deficiency in any of these proteins may result in the disassociation of the erythrocyte membrane structure and subsequently reduce the surface-to-volume ratio, which finally causes hereditary spherocytosis (Eber and Lux, 2004). Since ankyrin-1 encoded by ANK1 mediates the linkage of band-3 protein with spectrin, it plays an essential role in the stabilization of the membrane. Deficiency in ankyrin-1 protein may also result in the decreased assembly of spectrin, regardless of the spectrin synthesis (Hanspal et al., 1991).

In our case, the patient presented hereditary spherocytosis due to mutation in $A N K 1$. The proband was identified with heterozygous mutation c.2978T > A, which results in amino acid change p.I993N. Residue I993 is a highly conservative residue in ankyrin-1 protein among several species (Figure 3). The conservation of this residue indicates its importance in evolution. Besides, isoleucine is a non-polar neutral amino acid, while asparagine is a polar neutral amino acid. The alteration in polarity might change the interaction between amino acids within the structure or between ankyrin and other proteins, thus affecting the function of ankyrin. Moreover, residue resides in a domain called ZU5 (from residue 911 to residue 1,015). Experiment of Mohler et al. (2004) shows that this domain could help in the binding of spectrin. The occurrence of variation in this domain

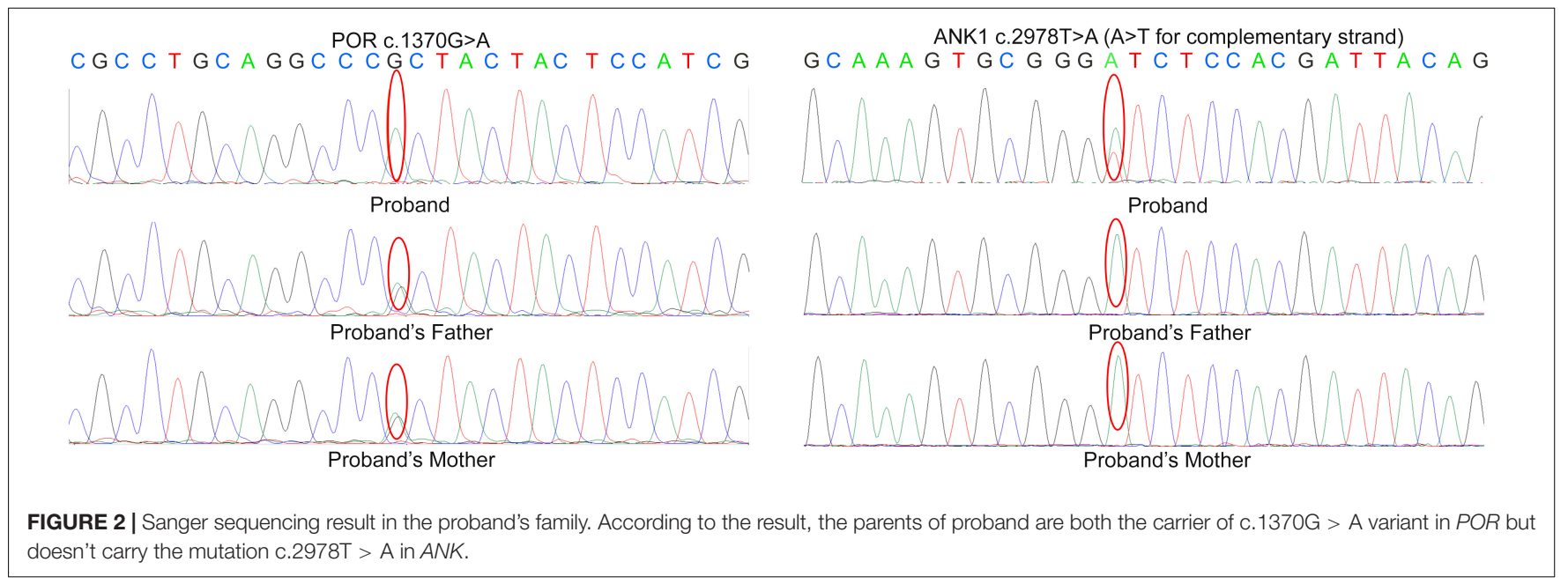




Human
Mouse
Chimpanzee
Zebrafish
Sheep
Southern_pig_tailed_macaque

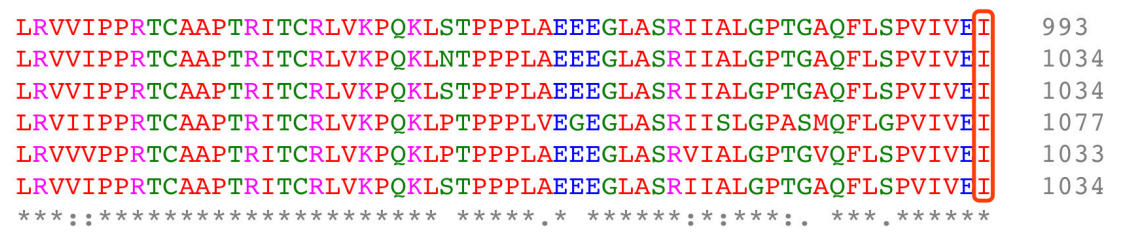

FIGURE 3 | Sequencing alignment of residue 1993 in ANK1 protein. Result shows that this residue is extremely conserved among species.

A

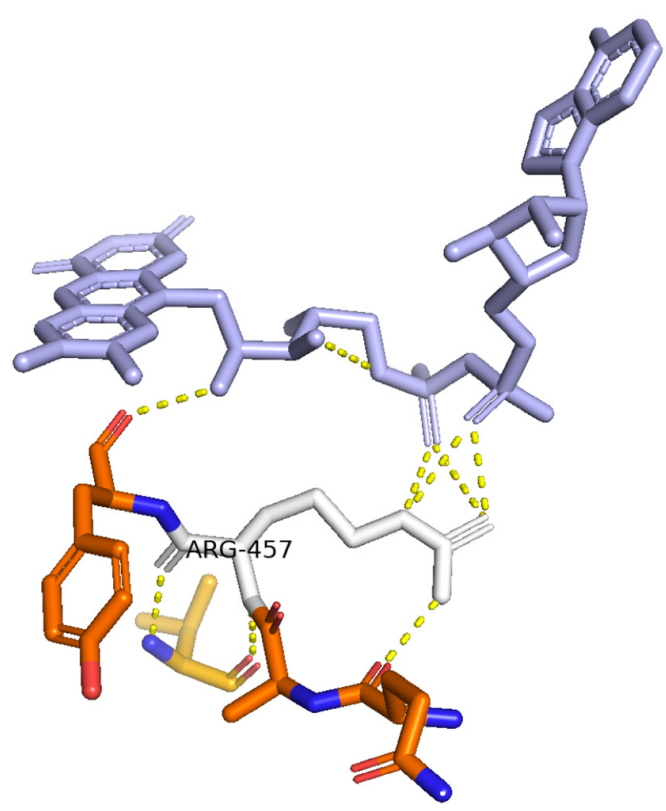

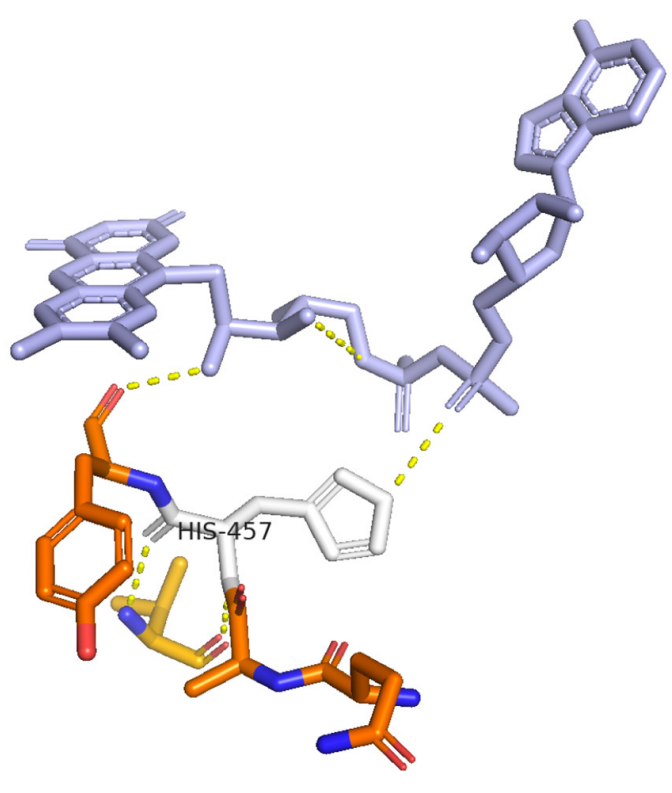

FIGURE 4 | Structure of cytochrome P450 oxidoreductase before and after mutation. (A) Crystal structure of wild-type P450 oxidoreductase (pbd id:5FA6). (B) Crystal structure of mutant P450 oxidoreductase (pdb id:3QFR). The attraction between P450 oxidoreductase and FAD was found significantly decreased.

TABLE 1 | Summarized clinical information of patients carrying mutation c.1370G > A (p.R457H) in the POR gene.

\begin{tabular}{|c|c|c|c|}
\hline Report & Sex & & Clinical Presentation \\
\hline Adachi et al. (2004) & $M$ & Com Het & $\begin{array}{l}\text { Cardiac arrest due to upper airway obstruction twice during infancy. Craniosynostosis, radiohumeral synostosis, carpal } \\
\text { and tarsal bone fusion, malformed and simple ear, arachnodactyly. }\end{array}$ \\
\hline Adachi et al. (2004) & $\mathrm{F}$ & Com Het & $\begin{array}{l}\text { Bilateral radiohumeral synostosis, midfacial hypoplasia, malformed ears, and genital ambiguity including clitoromegaly } \\
\text { and labial fusion. No secondary sex characteristics appeared at } 11 \text { years of age. }\end{array}$ \\
\hline Fukami et al. (2005) & $M$ & Com Het & $\begin{array}{l}\text { Craniosynostosis, midfacial hypoplasia, multiple digital joint contractures, arachnodactyly, scoliosis at } 13 \text { years of age, } \\
\text { micropenis, cryptorchidism, hypospadias. }\end{array}$ \\
\hline Fukami et al. (2005) & $M$ & Com Het & $\begin{array}{l}\text { Craniosynostosis, midfacial hypoplasia, radiohumeral synostosis at } 10 \text { years of age, multiple digital joint contractures, } \\
\text { arachnodactyly, micropenis, cryptorchidism. }\end{array}$ \\
\hline Fukami et al. (2005) & M & Com Het & Craniosynostosis, midfacial hypoplasia, multiple digital joint contractures, arachnodactyly, cryptorchidism. \\
\hline Fukami et al. (2005) & $\mathrm{F}$ & Hom & Craniosynostosis, midfacial hypoplasia, multiple digital joint contractures, clitoromegaly, labial fusion. \\
\hline Fukami et al. (2005) & $\mathrm{F}$ & Het & Craniosynostosis, midfacial hypoplasia, multiple digital joint contractures, arachnodactyly, labial fusion. \\
\hline Fukami et al. (2005) & $\mathrm{F}$ & Hom & Craniosynostosis, midfacial hypoplasia, multiple digital joint contractures, clitoromegaly, labial fusion. \\
\hline Fukami et al. (2005) & $\mathrm{F}$ & Com Het & $\begin{array}{l}\text { Craniosynostosis, midfacial hypoplasia, conduction deafness at } 13 \text { years of age, multiple digital joint contractures, } \\
\text { arachnodactyly, clitoromegaly, labial fusion. }\end{array}$ \\
\hline Fukami et al. (2005) & $\mathrm{F}$ & Hom & Proximal interphalangeal joint, clitoromegaly. \\
\hline Arlt et al. (2004) & $\mathrm{F}$ & Com Het & $\begin{array}{l}\text { Clubfeet, ambiguous genitalia, clitoromegaly, labial fusion, marfanoid habitus, scoliosis, arachnodactyly, dysplastic ears, } \\
\text { slim limbs, unilateral oophorectomy. }\end{array}$ \\
\hline Flück et al. (2004) & M & Com Het & $\begin{array}{l}\text { Craniosynostosis, hypertelorism, mild choanal narrowing, radiohumeral synostosis, midface hypoplasia, arachnodactyly, } \\
\text { rocker-bottom feet, clitoromegaly, labial fusion. }\end{array}$ \\
\hline Bai et al. (2017) & $\mathrm{F}$ & Hom & Labial fusion, ovarian cyst without special skeletal deformities. \\
\hline
\end{tabular}

Com Het, compound heterozygous; Hom, homozygous; Het, heterozygous. 
may disrupt the association between $\beta$-spectrin and ankyrin-1, thus dissociating the erythrocyte membrane structure.

Congenital adrenal hyperplasia refers to a series of disorders in adrenal steroidogenesis that can affect the biogenesis of glucocorticoid, mineralocorticoid, or sex steroid production. CAH consists of two forms: a severe form and a mild form. The severe form affects nearly one in 15,000 births worldwide, while the prevalence of the mild form is one in 1,000 or even higher in certain ethnic groups (Merke and Kabbani, 2001).

Enzymes involved in cortisol biosynthesis include 21-hydroxylase, $11 \beta$-hydroxylase, $17 \alpha$-hydroxylase, $3 \beta$-hydroxysteroid dehydrogenase (type II), steroidogenic acute regulatory protein, P450 cholesterol side-chain cleavage enzyme, and P450 oxidoreductase $(P O R)$. The initial two enzymes in cortisol biosynthesis, $17 \mathrm{OH}$ and $21 \mathrm{OH}$, are functionally active only when the electron transport cytochrome P450 oxidoreductase exists. Therefore, patients with $P O R$ deficiency present combined $17 \mathrm{OH}$ and $21 \mathrm{OH}$ deficiency. The patient in our study was identified as carrying homozygous mutation c.1370G > A, which caused amino acid variation p. $\mathrm{R} 457 \mathrm{H}$. This mutation is a reported variant that is located in the FAD binding domain. The guanidinium group of residue R457 was proven to form a salt bridge with the pyrophosphate group of FAD (Wang et al., 1997; Xia et al., 2011). Alteration in H457 weakens the interaction between FAD and POR according to the molecule structure model (Figure 4). Since this variant is a known disease-causing mutation, the clinical presentation is also summarized as Table 1 (Adachi et al., 2004; Arlt et al., 2004; Flück et al., 2004; Fukami et al., 2005; Bai et al., 2017). According to the summarized information, nearly all patients carrying mutation $\mathrm{R} 457 \mathrm{H}$ are diagnosed as having Antley-Bixler syndrome, except for one patient, who did not present any skeletal malformation. Our patient presented arachnodactyly, multiple digital contractures, scoliosis, and clitoromegaly, as in most of the reported patients; therefore, she could be diagnosed as having Antley-Bixler syndrome.

In this study, we reported a girl presenting both spherocytosis and Antley-Bixler syndrome with genital anomalies and disordered steroidogenesis. Patients presenting more than one diseases, especially hereditary diseases, are not a common affair for the clinicians. Therefore, when handling cases presenting multi-system symptoms, some of the clinicians would tend to treat it as a rare syndrome not several separated diseases. This thought might impede the analysis of genetic tests. We hope our experience in diagnosing this case would delight other clinicians in handling patients with disease presentations in multiple systems. Moreover, the cooperation between doctors in different department is also important. In our case, the clitoromegaly

\section{REFERENCES}

Adachi, M., Tachibana, K., Asakura, Y., Yamamoto, T., Hanaki, K., and Oka, A. (2004). Compound heterozygous mutations of cytochrome P450 oxidoreductase gene (POR) in two patients with Antley-Bixler syndrome. Am. J. Med. Genet. A 128a, 333-339. doi: 10.1002/ajmg.a.30169

Arlt, W., Walker, E. A., Draper, N., Ivison, H. E., Ride, J. P., Hammer, F., et al. (2004). Congenital adrenal hyperplasia caused by mutant P450 oxidoreductase of the patient was treated when she was 3 years old in other hospital without a clear diagnosis. She was admitted to the hematological department of our hospital at the age of 5 only due to the complaint of spherocytosis. However, since the patient was found to have arachnodactyly during admission she was then sent to the department of endocrinology. After the nextgeneration sequencing and the examination of clinicians in both departments, she was finally diagnosed.

\section{DATA AVAILABILITY STATEMENT}

The raw data supporting the conclusions of this article will be made available by the authors, without undue reservation.

\section{ETHICS STATEMENT}

The study was approved by the Ethics Committee of the Capital Institute of Pediatrics. Written informed consent was obtained from the patient's parents for the publication of this report and any accompanying images.

\section{AUTHOR CONTRIBUTIONS}

SF and RL were in charge of the diagnosis of spherocytosis and also the writing works concerning the presentations of spherocytosis. They also gave advices when doing the gene analysis. FS, MD, HY, and $\mathrm{XC}$ were responsible for the diagnosis of $\mathrm{CAH}$ and all the description parts of $\mathrm{CAH}$ in this article. They also gave advices when doing the gene analysis. XS took the charge of performing gene sequencing and the analysis and also the genetic parts of this article. All authors agreed to be accountable for the content of the work.

\section{ACKNOWLEDGMENTS}

We are grateful to all of the family members for their participation in the study.

\section{SUPPLEMENTARY MATERIAL}

The Supplementary Material for this article can be found online at: https://www.frontiersin.org/articles/10.3389/fgene. 2020.00976/full\#supplementary-material

and human androgen synthesis: analytical study. Lancet 363, 2128-2135. doi: 10.1016/s0140-6736(04)16503-3

Bai, Y., Li, J., and Wang, X. (2017). Cytochrome P450 oxidoreductase deficiency caused by $\mathrm{R} 457 \mathrm{H}$ mutation in POR gene in Chinese: case report and literature review. J. Ovarian Res. 10:16.

Eber, S., and Lux, S. E. (2004). Hereditary spherocytosis-defects in proteins that connect the membrane skeleton to the lipid bilayer. Semin. Hematol. 41, 118-141. doi: 10.1053/j.seminhematol.2004.01.002 
El-Maouche, D., Arlt, W., and Merke, D. P. (2017). Congenital adrenal hyperplasia. Lancet 390, 2194-2210.

Flück, C. E., Tajima, T., Pandey, A. V., Arlt, W., Okuhara, K., Verge, C. F., et al. (2004). Mutant P450 oxidoreductase causes disordered steroidogenesis with and without Antley-Bixler syndrome. Nat. Genet. 36, 228-230. doi: 10.1038/ ng1300

Fukami, M., Horikawa, R., Nagai, T., Tanaka, T., Naiki, Y., Sato, N., et al. (2005). Cytochrome P450 oxidoreductase gene mutations and Antley-Bixler syndrome with abnormal genitalia and/or impaired steroidogenesis: molecular and clinical studies in 10 patients. J. Clin. Endocrinol. Metab. 90, 414-426. doi: 10.1210/jc.2004-0810

Gallagher, P. G. (2005). Hematologically important mutations: ankyrin variants in hereditary spherocytosis. Blood Cells Mol. Dis. 35, 345-347. doi: 10.1016/ j.bcmd.2005.08.008

Hannah-Shmouni, F., Chen, W., and Merke, D. P. (2017). Genetics of Congenital Adrenal Hyperplasia. Endocrinol. Metab. Clin. North Am. 46, 435-458.

Hanspal, M., Yoon, S. H., Yu, H., Hanspal, J. S., Lambert, S., Palek, J., et al. (1991). Molecular basis of spectrin and ankyrin deficiencies in severe hereditary spherocytosis: evidence implicating a primary defect of ankyrin. Blood 77, 165-173. doi: 10.1182/blood.v77.1.165.165

Merke, D., and Kabbani, M. (2001). Congenital adrenal hyperplasia: epidemiology, management and practical drug treatment. Paediatr. Drugs 3, 599-611. doi: 10.2165/00128072-200103080-00005

Mohler, P. J., Yoon, W., and Bennett, V. (2004). Ankyrin-B targets beta2-spectrin to an intracellular compartment in neonatal cardiomyocytes. J. Biol. Chem. 279, 40185-40193. doi: 10.1074/jbc.m406018200

Perrotta, S., Gallagher, P. G., and Mohandas, N. (2008). Hereditary spherocytosis. Lancet $372,1411-1426$.
Wang, M., Roberts, D. L., Paschke, R., Shea, T. M., Masters, B. S., and Kim, J. J. (1997). Three-dimensional structure of NADPH-cytochrome P450 reductase: prototype for FMN- and FAD-containing enzymes. Proc. Natl. Acad. Sci. U.S.A. 94, 8411-8416. doi: 10.1073/pnas.94.16. 8411

Witchel, S. F. (2017). Congenital adrenal hyperplasia. J. Pediatr. Adolesc. Gynecol. 30, 520-534.

Xia, C., Panda, S. P., Marohnic, C. C., Martasek, P., Masters, B. S., and Kim, J. J. (2011). Structural basis for human NADPH-cytochrome P450 oxidoreductase deficiency. Proc. Natl. Acad. Sci. U.S.A. 108, 13486-13491. doi: 10.1073/pnas. 1106632108

Yocum, A. O., Steiner, L. A., Seidel, N. E., Cline, A. P., Rout, E. D., Lin, J. Y., et al. (2012). A tissue-specific chromatin loop activates the erythroid ankyrin-1 promoter. Blood 120, 3586-3593. doi: 10.1182/blood-2012-08450262

Conflict of Interest: XS was employed by the company Running Gene Inc.

The remaining authors declare that the research was conducted in the absence of any commercial or financial relationships that could be construed as a potential conflict of interest.

Copyright (c) 2020 Song, Feng, Shen, Du, Yin, Liu and Chen. This is an open-access article distributed under the terms of the Creative Commons Attribution License (CC BY). The use, distribution or reproduction in other forums is permitted, provided the original author(s) and the copyright owner(s) are credited and that the original publication in this journal is cited, in accordance with accepted academic practice. No use, distribution or reproduction is permitted which does not comply with these terms. 